\title{
Endothelial Dysfunction in Type 2 Diabetes Mellitus
}

\author{
R. Dhananjayan ${ }^{1}$ K. S. Srivani Koundinya ${ }^{2} \cdot$ T. $_{\text {Malati }}{ }^{3} \cdot$ Vijay Kumar Kutala $^{4}$
}

Received: 16 December 2014/ Accepted: 29 July 2015/Published online: 29 September 2015

(C) Association of Clinical Biochemists of India 2015

\begin{abstract}
Endothelial dysfunction is an imbalance in the production of vasodilator factors and when this balance is disrupted, it predisposes the vasculature towards prothrombotic and pro-atherogenic effects. This results in vasoconstriction, leukocyte adherence, platelet activation, mitogenesis, pro-oxidation, impaired coagulation and nitric oxide production, vascular inflammation, atherosclerosis and thrombosis. Endothelial dysfunction is focussed as it is a potential contributor to the pathogenesis of vascular disease in diabetes mellitus. Under physiological conditions, there is a balanced release of endothelial-derived relaxing and contracting factors, but this delicate balance is altered in diabetes mellitus and atherosclerosis, thereby contributing to further progression of vascular and endorgan damage. This review focuses on endothelial dysfunction in atherosclerosis, insulin resistance, metabolic syndrome, oxidative stress associated with diabetes mellitus, markers and genetics that are implicated in endothelial dysfunction.
\end{abstract}

Keywords Endothelial dysfunction - Type 2 diabetes mellitus · Oxidative stress $\cdot$ eNOS $\cdot$ IRS-1

R. Dhananjayan

arjunbio@gmail.com

1 Department of Biochemistry, ACS Medical College \& Hospital, Velappanchavadi, Chennai, Tamil Nadu, India

2 Meenakshi Academy of Higher Education (University), Chennai, Tamil Nadu, India

3 Department of Biochemistry, Nizam's Institute of Medical Sciences, Hyderabad, Telangana, India

4 Department of Clinical Pharmacology and Therapeutics (Biochemistry), Nizam's Institute of Medical Sciences, Hyderabad, Telangana, India

\begin{tabular}{|c|c|}
\hline \multicolumn{2}{|c|}{ Abbreviations } \\
\hline $\mathrm{ACh}$ & Acetyl choline \\
\hline AGE & Advanced glycosylation end-product \\
\hline Ang II & Angiotensin II \\
\hline AR & Aldose reductase \\
\hline CAD & Coronary artery disease \\
\hline cGMP & Cyclic guanosine monophosphate \\
\hline DAG & Diacylglycerol \\
\hline DM & Diabetes mellitus \\
\hline ECM & Extra cellular matrix \\
\hline ED & Endothelial dysfunction \\
\hline EDHF & Endothelial derived hyperpolarizing factor \\
\hline EDN1 & Endothelin-1 \\
\hline IRS1 & Insulin receptor substrate 1 \\
\hline eNOS & Endothelial nitric oxide synthase \\
\hline ET-1 & Endothelin-1 \\
\hline GFAT & $\begin{array}{l}\text { Glutamine fructose-6-phosphate } \\
\text { amidotransferase }\end{array}$ \\
\hline GP & Glycerol 3-phosphate \\
\hline GSH & Glutathione \\
\hline hsCRP & High sensitive C-reactive protein \\
\hline IMT & Intimal medial thickening \\
\hline MAPK & Mitogen activated protein kinase \\
\hline MCP-1 & Monocyte chemo-attractant protein-1 \\
\hline MCP-1 & Monocyte chemotactic protein-1 \\
\hline MDA & Malondialdehyde \\
\hline MTHFR & Methylene tetrahydrofolate reductase \\
\hline $\mathrm{NF}-\kappa \mathrm{B}$ & Nuclear factor $\kappa \mathrm{B}$ \\
\hline NO & Nitric oxide \\
\hline PA & Phosphatidic acid \\
\hline PAI-1 & Plasminogen activator inhibitor-1 \\
\hline PG & Prostaglandin \\
\hline PGI & Prostacyclin \\
\hline PIK3 & Phosphatidyl inositol-3'-kinase \\
\hline PUFA & Polyunsaturated fatty acid \\
\hline
\end{tabular}


ROS Reactive oxygen species

SDH Sorbitol dehydrogenase

SNP Single nucleotide polymorphism

t-PA Tissue-type plasminogen activator

VCAM-1 Vascular cell adhesion molecule-1

VSMC Vascular smooth muscle cell

vWF Von Willebrand factor

\section{Introduction}

Endothelium is the biological active inner layer of the blood vessels, which controls vascular and thus organ functions. The arterial vessel is outlined by three distinct layers; tunica intima-a single layer of endothelial cells, tunica media-which comprises the vascular smooth muscle cell (VSMC) and tunica adventitia, an elastic lamina with terminal nerve fibres and surrounding connective tissue. The endothelium plays an important role in vascular homeostasis by modulating blood flow, delivery of nutrients, VSMC proliferation and migration, fibrinolysis and coagulation, inflammation, platelet and leukocyte adherence [1]. To carry out the above functions, the endothelium produces components of the ECM such as collagen and a variety of regulatory mediators, including nitric oxide (NO), prostanoids, endothelin-1 (ET-1), angiotensin II (Ang II), tissue-type plasminogen activator (t-PA), plasminogen activator inhibitor-1 (PAI-1), von Willebrand factor (vWF), adhesion molecules and cytokines. The production of these moieties is responsible for various stimuli [2]. Normal endothelium actively decreases vascular tone, limits leukocyte adhesion and thus inflammatory activity in the vessel wall, maintains vascular permeability to nutrients, hormones, other macromolecules and leucocytes, inhibits platelet adhesion and aggregation by producing prostacyclin and $\mathrm{NO}$, limits activation of the coagulation cascade by the thrombomodulin/protein C, heparan sulphate/antithrombin and tissue factor/tissue factor pathway inhibitor interactions, regulates fibrinolysis by producing t-PA and its inhibitor PAI-1.

Endothelial cell constitutively express endothelial nitric oxide synthase (eNOS) that after $\mathrm{Ca}^{2+} /$ calmodulin binding generates $\mathrm{NO}$ using L-arginine as a substrate together with cofactors, e.g., NADPH and tetrahydrobiopterin. NO then rapidly diffuses into VSMC and binds to a heme group of soluble guanylate cyclase. This event results in the formation of cyclic guanosine monophosphate (cGMP), activating a cGMP dependent protein kinase, which leads to an increased extrusion of $\mathrm{Ca}^{2+}$ from cytosol in VSMC, inhibiting the contractile machinery and there by initiating vasodilation [3]. The production and release of NO may be further increased by circulating factors, such as acetyl choline (ACh), bradykinin and serotonin. NO is also released by physical stimuli such as shear stress and ischemia which is not $\mathrm{Ca}^{2+} /$ calmodulin dependent. Apart from vasodilator effect, NO inhibits expression of proinflammatory cytokines, chemokines and leukocyte adhesion molecules, thereby limiting vascular recruitment of leukocytes and platelets. It also inhibits VSMC proliferation, an early sign of atherosclerosis [4]. Therefore, the NO synthesized by endothelial cells is an important endothelium-derived mediator, because of its vasodilator, antiplatelet, anti-proliferative, permeability-decreasing and anti-inflammatory properties [5]. NO inhibits leucocyte adhesion and rolling as well as cytokine-induced expression of vascular cell adhesion molecule-1 (VCAM-1) and monocyte chemotactic protein-1 (MCP-1) [6], effects that are at least in part attributable to inhibition of the transcription factor nuclear factor $\kappa \mathrm{B}(\mathrm{NF}-\kappa \mathrm{B})$ [7].

The endothelial dysfunction (ED) is an imbalance in the production of vasodilator factors, e.g., NO, prostacyclin $\left(\mathrm{PGI}_{2}\right)$, endothelial derived hyperpolarizing factor (EDHF) and vasoconstricting factors, e.g., ET-1, Ang-II and prostaglandin (PGH2). When this balance is disrupted, it predisposes the vasculature towards pro-thrombotic and proatherogenic effects. This results in vasoconstriction, leukocyte adherence, platelet activation, mitogenesis, prooxidation, impaired coagulation, vascular inflammation, atherosclerosis and thrombosis [8].

In the presence of suboptimal concentrations of substrate or cofactors for the synthesis of NO, eNOS may become uncoupled, resulting in the production of reactive oxygen species (ROS). At high concentrations of ROS, the scavenging system is impeded and NO may rapidly react with superoxide species to form peroxynitrite, exaggerating the oxidative stress further. The molecular cause of oxidative stress may be due to hyperglycemia, dyslipidemia, cigarette smoking, inflammation and insulin resistance. Many studies demonstrate that the loss of biological activity and/or biosynthesis of $\mathrm{NO}$ are the molecular basis of ED [9].

The endothelium has a limited intrinsic capacity of selfrepair, being built up by terminally differentiated cells with a low proliferative potential. Therefore the endothelial repair is accomplished through the contribution of circulating cells, namely endothelial progenitor cells (EPCs), in physiological and pathological conditions. An earlier study states that EPCs has a central role in the development and progression of virtually all diabetic complications [10]. A key mechanism of diabetic vascular disease is the development of ED, which is characterized by a loss of NO and development of a proinflammatory vascular phenotype that promotes atherosclerosis and cardiovascular events [11]. Another study reported that continuous intravenous 
infusion of glucagon-like peptide-1 (GLP-1) resulted in short-term, blood glucose-independent improvement of vascular ED [12]. It has been reported that GLP-1 receptors are expressed in vascular endothelial cells and it has been reported to directly increase NO production and inhibit the expressions of endothelial cell adhesion factors [13].

\section{Endothelial Dysfunction in Type 2 Diabetes Mellitus}

ED is focussed as it is a potential contributor to the pathogenesis of vascular disease in DM. Under physiological conditions, there is a balanced release of endothelial-derived relaxing and contracting factors, but this delicate balance is altered in DM and atherosclerosis, thereby contributing to further progression of vascular and end-organ damage [14]. Endothelium plays an important role in regulating vascular tone and structure. A healthy endothelium inhibits platelet and leukocyte adhesion to the vascular surface and maintains a balance of profibrinolytic and prothrombotic activity [15]. Hyperglycemia is the major factor in the development of ED in DM. Insulin resistance has been described in several diseases that increase cardiovascular risk and mortality, such as DM, obesity, hypertension, metabolic syndrome and heart failure. Increasing evidence suggests that the progression of insulin resistance to $\mathrm{T} 2 \mathrm{D}$ parallels the progression of $\mathrm{ED}$ to atherosclerosis.

ED in diabetes originates from the following sources, (1) hyperglycaemia and its immediate biochemical complications directly alter endothelial function; (2) high glucose influences endothelial cell functioning indirectly by the synthesis of growth factor and vasoactive agents in other cells; (3) the components of the metabolic syndrome. In normal conditions, glucose is metabolized through the glycolytic pathway. An increase in intracellular glucose leads to an increase in four pathways: the flux of glucose to sorbitol via the sorbitol pathway, an increase in fructosamine 6-phosphate via the hexosamine pathway, the activation of protein kinase-C (PKC) via de novo synthesis of diacylglycerol (DAG) and the formation of advanced glycosylation end-product (AGE) (Fig. 1). Excess glucose can be metabolized in the sorbitol pathway to sorbitol and fructose by aldose reductase (AR) and sorbitol dehydrogenase (SDH). In hexosamine pathway, fructose 6-phosphate is converted into fructosamine 6-phosphate by the enzyme glutamine fructose-6-phosphate amidotransferase (GFAT) and subsequently into GlcNAc. The mechanism responsible for the activation of $\mathrm{PKC}$ by hyperglycaemia is related to de novo synthesis of the PKC activator, DAG from a stepwise acylation of glycerol 3-phosphate (GP) and phosphatidic acid (PA). The above four biochemical and metabolic mechanisms are the consequence of a hyperglycaemia induced overproduction of oxidative stress in the mitochondria. In addition to this pathway, hyperglycaemia may lead to increased reactive oxygen species (ROS) by activation of NADPH oxidase, inactivation and reduced expression of the antioxidant enzymes, catalase and superoxide dismutase (SOD) or uncoupling of endothelial nitric oxide synthase (eNOS) [16].

\section{Endothelial Dysfunction and Atherosclerosis}

Atherosclerosis is a condition in which an artery wall thickens as a result of the accumulation of cholesterol and triglycerides and the hardening of the arteries is caused by the formation of multiple plaques within the arteries. Atherogenesis is the deleterious alterations of endothelial physiology, an early step in the development of atherosclerosis and also involve in plaque progression and occurrence of atherosclerotic complications. Most of the risk factors related to atherosclerosis and cardiovascular morbidity and mortality, are found to be associated with ED. Many of these risk factors, including hyperlipidemia, hypertension, diabetes and smoking are associated with overproduction of ROS or increased oxidative stress [17].

In the atherosclerotic process, ED is a key factor which precedes and creates a vulnerable environment in the arteries. During advanced ED in T2D, leukocytes and other inflammatory cells infiltrate the intimal layer of the endothelium [18]. Abnormalities in platelet function and coagulation are also commonly seen in T2D patients. The angiopathic processes are exaggerated in $\mathrm{T} 2 \mathrm{D}$, associated with increased inflammation and intraplaque haemorrhage. Macrophage infiltration is increased in plaques and procoagulant factors are up-regulated in T2D patients. These differences in plaque composition, coagulation, platelet function and inflammation may contribute to the advanced atherosclerosis seen in T2D [19].

\section{Endothelial Dysfunction and Insulin Resistance}

Insulin resistance is defined as the decreased ability of insulin to promote glucose uptake in skeletal muscle and adipose tissue. To suppress hepatic glucose output, may be present for many years before the development of any abnormality in plasma glucose levels [20, 21]. Insulin resistance syndrome encompasses more than a subnormal response to insulin-mediated glucose disposal. Patients with this syndrome have elevated blood pressure, hyperlipidemia and dysfibinolysis even without any clinically demonstrable alteration in plasma glucose concentrations. Insulin resistance may be linked to ED by a number of 


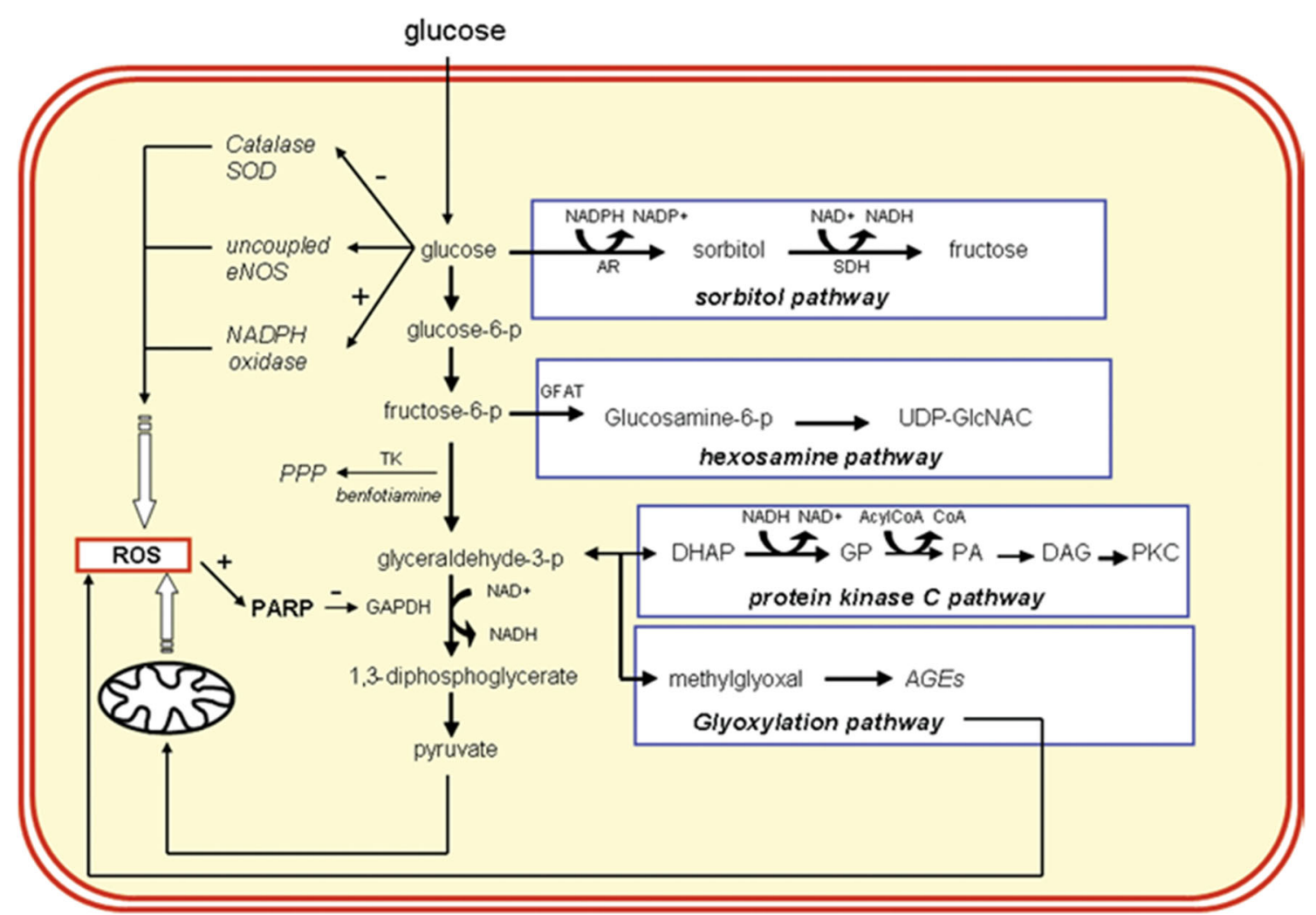

Fig. 1 Hyperglycemia and endothelial dysfunction [62]

mechanisms, including disturbances of sub cellular signalling pathways common to both insulin action and NO production. Other potential unifying links include the roles of oxidative stress, endothelin, renin angiotensin system, the secretion of hormones and cytokines by adipose tissue. Individuals who advance toward the development of T2D experience progressive deterioration of glucose tolerance over time. Obesity is also has an important genetic component, invariably exacerbates any degree of insulin resistance [19]. Obesity and insulin resistance are usually present for many years before the appearance of other abnormalities such as hypertension, dyslipidemia, T2D and CVD. In some individuals, obesity and insulin resistance may be present during childhood and adolescence [21].

Multiple, interrelated mechanisms contribute to ED in insulin resistance. It is known that eNOS infiltrates into caveolae, which are cholesterol-rich invaginations present in endothelial cells and VSMC that decrease vasoconstrictive responses to Ang-II, endothelin and constitutive eNOS activity in animals [22]. Addition of oxidized LDL-c to cultured endothelial cells disrupts the caveolae complex and is thought to be associated with decreased eNOS activity and ED [23, 24]. HDL-c can prevent the oxidized LDL-mediated decrease in cholesterol in caveolae, prevent the translocation of eNOS and caveolin from caveolae, and prevent the decrease in responsiveness to Ach [25]. These effects occur because HDL-c donates cholesterol to the caveolae complex. These cellular events are consistent with the pro-atherogenic effects of LDL-c, oxidized LDL-c and the protective effects of HDL-c. The presence of hypertension and other atherosclerotic risk factors is associated with increased vascular Ang-II generation and activity [26]. Because Ang-II and insulin activate a common signalling pathway, increased sensitivity to Ang-II may occur in the hyperinsulinemic, insulin-resistant state. In addition, Ang-II stimulates ICAM-1 and monocyte chemo-attractant protein-1 (MCP-1) through the mitogen activated protein kinase (MAPK) pathway in endothelial cells and VSMC [27].

\section{Endothelial Dysfunction and Metabolic Syndrome}

Hypertension, dyslipidemia and hyperglycemia were noted as resistant to insulin stimulated glucose uptake and the changes associated with this comprise a syndrome known as metabolic syndrome. WHO defined metabolic syndrome as insulin resistance/IGT/IFG/diabetes together with two of the components, hypertension, hypertriglyceridemia, low LDL-c, central obesity and microalbuminuria. The risk 
factors or metabolic syndrome increase the risk for CVD along with T2D [28, 29]. The initiation and progression of atherosclerosis may have its origins in impaired endothelial function that can be detected at early stages of development of the syndrome. The basic elements of the metabolic syndrome and accelerated phase of atherogenesis are often silent partners that present many years before the onset of T2D [30]. Over time, insulin resistance is associated with more components of the metabolic syndrome including, low HDL-c level, hypertension, increased vascular production of ROS, increased plasma PAI-1-mediated thrombotic tendency, hyperuricemia, high triglyceride levels and elevation of oxidation-prone LDL-c levels [31].

\section{Oxidative Stress and Endothelial Dysfunction}

Diabetic endothelium produces an increase in both $\mathrm{O}_{2}^{-}$and $\mathrm{H}_{2} \mathrm{O}_{2}$ leading to enhanced intracellular production of $\mathrm{OH}$. Thus $\mathrm{OH}^{-}$is implicated in diabetes-induced endothelial dysfunction [32]. ROS are generated at sites of inflammation and injury. ROS at low concentrations can function as signalling molecules participating as signalling intermediates in the regulation of fundamental cell activities such as cell growth and cell adaptation responses, whereas at higher concentrations, ROS can cause cellular injury and death. The vascular endothelium, which regulates the passage of macromolecules and circulating cells from blood to tissues, is a major target of oxidative stress, playing a critical role in the pathophysiology of several vascular diseases and disorders. Specifically, oxidative stress increases vascular endothelial permeability and promotes leukocyte adhesion, which is coupled with alterations in endothelial signal transduction and redox-regulated transcription factors [33]. Decreased endothelium-dependent vasodilation in diabetic subjects is associated with the impaired action of NO secondary to its inactivation resulting from increased oxidative stress, rather than decreased NO production from vascular endothelium, and that abnormal NO metabolism is related to advanced diabetic microvascular complications [34]. Endogenous ROS in high quantities overwhelm the innate antioxidant defense system leading to oxidative stress. Oxidative stress appears to be involved in aging and various diseases, including DM [35]. The glucolipotoxicity is complicated with endothelial dysfunction and susceptible to oxidative stress, as higher blood glucose level is associated with free radical-mediated lipid peroxidation [36].

Oxygen free radicals in DM can cause peroxidative breakdown of phospholipids that lead to accumulation of malondialdehyde (MDA). It plays a key role in modifying LDL-c, which mediates the pathophysiological changes by non-enzymatic and auto-oxidative glycosylation [37]. Cigarette smokers have elevated levels of oxidized macromolecules owing to increased ROS production and may act as an additional risk factor in the complications of DM [38]. The distressed equilibrium between pro-oxidants and antioxidants in DM alters the metabolic status of body, leading to development of microvascular and macrovascular complications that may increase with duration of the disease.

Glutathione, the tripeptide, $\gamma$-L-glutamyl-L-cysteinylglycine (GSH), is an antioxidant molecule synthesised in almost all living cells. GSH is able to protect cells from oxidation by virtue of the reducing power of the thiol group on the cysteine portion of the molecule. A consistent finding in T2D is a high free radical load, which is associated with the hyperglycemia. The elevated glucose binds to proteins, glycating them and leads to the formation of advanced glycation end products (AGEs) and many free radicals [39]. Advanced glycation end products are associated with tissue damage and aging [40]. The glycation of proteins in DM increases the rate of free radical production by nearly 50 -fold, and the generated free radicals were found to increase peroxidation of polyunsaturated fatty acids (PUFAs) in the cell membrane nearly twofold over control levels [41]. The less well the diabetes is controlled, i.e., the higher the blood glucose concentration, the more likely is the formation of glycated proteins. Glycated haemoglobin (HbA1c) is routinely measured in T2D, because once formed, it remains for the lifetime of the erythrocytes (120 days) and therefore acts as a reliable monitor of blood glucose control over 4 months. Glycation of haemoglobin of less than $6 \%$ is normal. HbAlc is more negatively charged than haemoglobin and has a higher oxygen affinity therefore reducing gaseous exchange at the tissues [42]. A decrease in GSH concentration in the erythrocytes of T2D patients is widely reported and this depletion of GSH is considered to be indicative of increased oxidative stress. However, many authors report unchanged or elevated GSH concentrations in the erythrocytes of T2D patients [43].

\section{Serum Markers of Endothelial Dysfunction}

Generally the substances released by endothelium have important autocrine as well as paracrine functions and help to maintain the normal health of vascular wall, haemostatic functions and hemodynamic balance of the entire body [15]. There are many clinical markers proposed to link inflammation and atherosclerosis. ED or damage can be approximated through measurement of serum biomarkers, such as ET-1, vWF, t-PA, PAI-1, ICAMs, VCAMs, E-selectin, P-selectin, high sensitive C-reactive protein (hSCRP) and $\mathrm{NO}$ which are released from endothelial cells.

It is perceived that chronic low-grade inflammation as evidenced by elevated hsCRP might potentially be a cause 
underlying the etiology and manifestation of T2D. Circulating hsCRP is a strong predictor of cardiovascular death [44]. CRP is released by the cytokines IL-1, TNF- $\alpha$ and IL- 6 from the liver as acute phase reactant in response to inflammation. It may directly promote atherosclerosis and endothelial inflammation [12]. It may directly trigger the development of a pro-inflammatory and pro-atherosclerotic state, leading to atherothrombosis [45]. The in vitro data, largely in endothelial cells, but also in monocytes/macrophages and vascular smooth muscle cells, have suggested a role for CRP in atherogenesis [46]. The pro-inflammatory, pro-atherogenic effects of CRP that have been documented in endothelial cells include the following: decreased NO and prostacyclin and increased ET-1, cell adhesion molecules, MCP-1, IL-8 and PAI-1. In monocytes/macrophages, it induces tissue factor secretion, increases ROS and pro-inflammatory cytokine release, promotes monocyte chemotaxis and adhesion and increases oxidized LDL-c uptake. CRP has also been shown in vascular smooth muscle cells to increase inducible NO production, increase $\mathrm{NF}-\kappa \mathrm{B}$ and MAPK activities and most importantly, up-regulate Ang II type 1 receptor, resulting in increased ROS and vascular smooth muscle cell proliferation.

$\mathrm{NO}$ is produced by the conversion of L-arginine to $\mathrm{L}-$ citrulline by eNOS. NOS is activated, upon stimulation of endothelium by a variety of stimuli including shear stress, substance $\mathrm{P}$ and acetyl choline. NO, thus formed diffuses into the underlying vascular smooth muscles to stimulate the activation of guanylate cyclase, which in turn converts GTP to the smooth muscle relaxant compound cGMP in the vascular smooth muscle cells [47]. The increase in cGMP leads to activation of cyclic GMP-dependent protein kinase, which in turn leads to phosphorylation of potassium dependent channels with consecutive hyper polarization and extrusion of calcium ions resulting in VSMC relaxation [48].

\section{Genetics of Endothelial Dysfunction}

Single nucleotide polymorphisms (SNPs) are small genetic changes or variations of a single base found in the sequence of DNA and are defined as polymorphisms when they are present at $1 \%$ or more within a population. The frequency of each SNP is highly variable between different populations within a single species. It is well known that genetic factors contribute to the appearance and development of these chronic micro- and macro-angiopathic complications, but environmental factors usually trigger their appearance. Among these factors, as a product of hyperglicemia, ROS generate endothelial damage. The development of T2D requires the involvement of genetic and environmental factors such as obesity and sedentary lifestyle that determine hyposecretion of insulin in response to glucose stimulation and a decreased insulin action in peripheral tissues. Even the more common forms of atherosclerosis manifest in later life, they are partly heritable, arising as a result of common environmental exposures (risk factors) and many common gene variants (polymorphisms) with small to moderate effect [49].

The vessels in the offspring or relatives of patients with premature CAD show structural and functional changes more commonly, even before clinically manifestations start [50]. Therefore, individual differences in endothelial function are susceptible to later atherosclerosis, might relate not only to different levels of exposure to risk factors, but also to inter-individual differences in the carriage of risk alleles of genes expressed in the vascular endothelium. The atheroprotective actions of endothelial mediators such as NO suggest that genes that regulate synthesis and inactivation of these mediators might be important. The products of various gene SNPs implicated in ED are associated with endothelium dependent vasoreactivity. These include angiotensin converting enzyme, methylene tetrahydrofolate reductase (MTHFR), neuropeptide $\mathrm{Y}$, the bradykinin receptor, IL-6, osteoprotogerin, glutathione cysteine ligase and the G-protein b3-subunit. The SNP of the following genes are associated with endothelial dysfunction and CAD, i.e., Connexin-37 (GJA4, gap junction component in endothelium and other tissues), eNOS, Endothelin-1 (EDN1, vasoconstrictor produced by vascular endothelial cells), E-selectin (involves in adhesion of leukocytes to vascular endothelial cells), IRS-1 (substrate of insulin receptor tyrosine kinase), IL-6 (promotion and regulation of acute inflammatory response), IL-10 (anti-inflammatory cytokine that inhibits cytotoxic activity), thrombomodulin (THBD, vascular endothelial cell surface receptor for thrombin), TNF- $\alpha$ (pro-inflammatory cytokine).

Studies have provided evidence for altered NO metabolism and impaired endothelial function in diabetes, probably due to polymorphisms in the eNOS gene [51]. NO is synthesized by a family of NOS enzymes in which three isoforms have been identified: two constitutive, the neuronal NOS (nNOS or NOS-1) and endothelial NOS (eNOS or NOS-3) and one inducible NOS (iNOS or NOS-2). eNOS is the main contributor of circulating NO [52]. Each isoform is coded by separate genes with a different pattern of expression [53]. eNOS gene is located on chromosome 7 and it comprises 26 exons and 25 introns, with an entire length of $21 \mathrm{~kb}$. Variants of eNOS gene contribute to ED and attenuate the NO production. Dysfunctional eNOS may play a critical role in the pathogenetic pathway, leading to diabetic vascular complications [54]. Several polymorphisms of the eNOS gene have been identified, and their association with various diseases has been investigated, 
including CAD, MI, coronary spasm, hypertension, endstage renal disease (ESRD) and diabetic nephropathy [55].

IRS- 1 is a protein that in humans is encoded by the IRS-1 gene. It plays a key role in transmitting signals from insulin and insulin-like growth factor-1 receptors to intracellular pathways PIK3 and MAPK pathways. PIK3 cascade controls IGF-1 mediated cell growth and survival [56]. IRS-1 gene has been proposed as having a role in the insulin-resistant disorders. The genetic analysis of the IRS-1 gene has revealed several base-pair changes that result in amino acid substitutions. Earlier studies have indicated that the presence of a mutated IRS-1 gene is associated with dyslipidemia, further suggesting that this gene variant may have a significant effect on several risk factors for CAD [57]. The naturally occurring mutations in IRS-1 have been identified to cause severe insulin resistance and diabetes. IRS-1, a major substrate for the insulin receptor tyrosine kinase is a candidate gene for inherited defects that predispose to diabetes. The most common amino acid change is a glycine to arginine substitution at codon 972 (G972R variant) has been implicated in both abnormal metabolic actions of insulin as well as defective insulin secretion [58, 59]. Earlier study demonstrated that the presence of the Arg972 allele is associated with lower IMT values of the carotid arteries [60].

In our earlier study, we have found that intimal medial thickening (IMT) of carotid artery was significantly higher in T2D patients and also increased with age and family history. The increased levels of lipids, hsCRP, IMT and decreased nitrite levels might contribute to the risk of ED in patients with T2D [61].

\section{Conclusion}

T2D is one of the major risk factor for ED and atherosclerosis which is presumably due to physiological, environmental factors (inflammation, age and hypertension) and genetic factors (SNPs of eNOS and IRS-1). Polymorphisms may affect crucial processes such as synthesis, repair and methylation of DNA; induce hyperhomocysteinemia; influence GSH levels and increase in oxidative stress. These cellular and biochemical changes might trigger atherosclerosis in T2D and thus inflating CAD risk.

\section{References}

1. Szmitko PE, Wang CH, Weisel RD, de Almeida JR, Anderson TJ, Verma S. New markers of inflammation and endothelial cell activation: part I. Circulation. 2003;108:1917-23.

2. Quyyumi AA. Endothelial function in health and disease: new insights into the genesis of cardiovascular disease. Am J Med. 1998;105:32S-9S.
3. Mombouli JV, Vanhoutte PM. Endothelial dysfunction: from physiology to therapy. J mol cell Cardiol. 1999;31:61-74.

4. Furchgott RF. Albert Lasker medical research awards the discovery of endothelium-derived relaxing factor and its importance in the identification of nitric oxide. JAMA. 1996;276:1186-8.

5. Kawashima $\mathrm{S}$. The two faces of endothelial nitric oxide synthase in the pathophysiology of atherosclerosis. Endothelium. 2004;11:99-107.

6. Khan BV, Harrison DG, Olbrych MT, Alexander RW, Medford RM. Nitric oxide regulates vascular cell adhesion molecule 1 gene expression and redox-sensitive transcriptional events in human vascular endothelial cells. Proc Natl Acad Sci USA. 1996;93:9114-9.

7. Janssen-Heininger YM, Poynter ME, Baeuerle PA. Recent advances towards understanding redox mechanisms in the activation of nuclear factor kB. Free Radic Biol Med. 2000;28:1317-27.

8. Verma S, Anderson TJ. Fundamentals of endothelial function for the clinical cardiologist. Circulation. 2002;105:546-9.

9. Harrison DG. Cellular and molecular mechanisms of endothelial cell dysfunction. J Clin Invest. 1997;100:2153-7.

10. Avogaro A, Albiero M, Menegazzo L, Kreutzenberg SD, Fadini GP. Endothelial dysfunction in diabetes - the role of reparatory mechanisms. Diabetes Care. 2011;34(2):285-90.

11. Tabit CE, Chung WB, Hamburg NM, Vita JA. Endothelial dysfunction in diabetes mellitus: molecular mechanisms and clinical implications. Rev Endocr Metab Disord. 2010;11(1):61-74.

12. Nystrom T, Gutniak MK, Zhang Q, Zhang F, Holst JJ, Ahren B, et al. Effects of glucagon-like peptide-1 on endothelial function in type 2 diabetes patients with stable coronary artery disease. Am J Physiol Endocrinol Metab. 2004;287:1209-15.

13. Oyama J, Higashi Y, Node K. Do incretins improve endothelial function? Cardiovasc Diabetol. 2014;13:21.

14. Tan KC, Chow WS, Tam SC, Ai VH, Lam CH, Lam KS, et al. Atorvastatin lowers C-reactive protein and improves endothelium-dependent vasodilation in type 2 diabetes mellitus. J Clin Endocrinol Metab. 2002;87:563-8.

15. Libby P, Ridker PM, Maseri A. Inflammation and atherosclerosis. Circulation. 2002;105(9):1135-43.

16. Brownlee M. Biochemistry and molecular cell biology of diabetic complications. Nature. 2001;414:813-20.

17. Kinlay S, Ganz P. Role of endothelial dysfunction in coronary artery disease and implications for therapy. Am J Cardiol. 1997;80(9A):11I-6I.

18. Trichon BH, Roe MT. Diabetes mellitus and ischemic heart disease. In: Marso SP, Stern DM, editors. Diabetes and cardiovascular disease integrating science and clinical medicine. Philadelphia: Lippincott Williams \& Wilkins; 2004. p. 355-78.

19. Kahn BB, Flier JS. Obesity and insulin resistance. J Clin Invest. 2000;106:473-81.

20. Haffner SM. Pre-diabetes, insulin resistance, inflammation and CVD risk. Diabetes Res Clin Pract. 2003;61(Suppl 1):S9-18.

21. Goran MI, Ball GD, Cruz ML. Obesity and risk of type 2 diabetes and cardiovascular disease in children and adolescents. J Clin Endocrinol Metab. 2003;88:1417-27.

22. Rizzo V, McIntosh DP, Oh P, Schnitzer JE. In situ flow activates endothelial nitric oxide synthase in luminal caveolae of endothelium with rapid caveolin dissociation and calmodulin association. J Biol Chem. 1998;273:34724-9.

23. Blair A, Shaul PW, Yuhanna IS, Conrad PA, Smart EJ. Oxidized low-density lipoprotein displaces endothelial nitric-oxide synthase (eNOS) from plasmalemmal caveolae and impairs eNOS activation. J Biol Chem. 1999;274:32512-9.

24. Drab M, Verkade P, Elger M. Loss of caveolae, vascular dysfunction and pulmonary defects in caveolin-1 gene-disrupted mice. Science. 2001;293:2449-52. 
25. Uittenbogaard A, Shaul PW, Yuhanna IS, Blair A, Smart EJ. High-density lipoprotein prevents oxidized low-density lipoprotein-induced inhibition of endothelial nitric-oxide synthase localization and activation in caveolae. J Biol Chem. 2000;275: 11278-83.

26. Dzau VJ. Tissue angiotensin and pathobiology of vascular disease: a unifying hypothesis. Hypertension. 2001;37:1047-52.

27. Tummala PE, Chen XL, Sundell CL. Angiotensin II induces vascular cell adhesion molecule-1 expression in rat vasculature: a potential link between the renin angiotensin system and atherosclerosis. Circulation. 1999;100:1223-9.

28. World Health Organization. Definition, diagnosis and classification of diabetes mellitus and its complications, part 1: diagnosis and classification of diabetes mellitus. Report of a WHO Consultation Geneva 1999, p. 43-46.

29. Grundy SM, Brewer HB Jr., Cleeman JI, Smith SC Jr., Lenfant C. Definition of metabolic syndrome: report of the National Heart, Lung and Blood Institute/American Heart Association conference on scientific Issues related to definition. Circulation. 2004; 109:433-438.

30. McVeigh GE, Cohn JN. Endothelial dysfunction and the metabolic syndrome. Curr Diab Rep. 2003;3:87-92.

31. Toikka JO, Ahotupa M, Viikari JSA, Niinikoski H, Taskinen MT, Irjala $\mathrm{K}$, et al. Constantly low HDL cholesterol concentration relates to endothelial dysfunction and increased in vivo LDLoxidation in healthy young men. Atherosclerosis. 1999;147: 133-8.

32. Pieper GM, Langenstroer P, Siebeneich W. Diabetic-induced endothelial dysfunction in rat aorta: role of hydroxyl radicals. Cardiovasc Res. 1997;34:145-56.

33. Hazel L, Kenneth A, Roebuck O. Oxidant stress and endothelial cell dysfunction. Am J Physiol Cell Physiol. 2001;280:C719-41.

34. Maejima K, Nakano S, Himeno M, Tsuda S, Makiishi H, Ito T, et al. Increased basal levels of plasma nitric oxide in Type 2 diabetic subjects relationship to microvascular complications. J Diabetes Complic. 2001;15:135-43.

35. Ceriello A, Motz E. Is oxidative stress the pathogenic mechanism underlying insulin resistance, diabetes and cardiovascular disease? The common soil hypothesis revisited. Arterioscler Thromb Vasc Biol. 2004;24:816-23.

36. Su Y, Liu XM, Sun YM, Jin HB, Fu R, Wang YY, et al. The relationship between endothelial dysfunction and oxidative stress in diabetes and prediabetes. Int J Clin Pract. 2008;62:877-82.

37. Peerapatdit T, Patchanans N, Likidlilid A, Poldee S, Sriratanasathavorn C. Plasma lipid peroxidation and antioxidant nutrients in type 2 diabetic patients. J Med Assoc Thai. 2006;89:S147-55.

38. Bloomer RJ. Decreased blood antioxidant capacity and increased lipid peroxidation in young cigarette smokers compared to nonsmokers: impact of dietary intake. Nutr J. 2007;6:39.

39. Keenoy BMY, Vertommen J, De Leeuw I. Divergent effects of different oxidants on glutathione homeostasis and protein damage in erythrocytes from diabetic patients: effects of high glucose. Mol Cell Biochem. 2001;225:59-73.

40. Wautier JL, Schmidt AM. Protein glycation: a firm link to endothelial cell dysfunction. Circ Res. 2004;95:233-8.

41. Mullarkey CJ, Edelstein D, Brownlee M. Free radical generation by early glycation products: a mechanism for accelerated atherogenesis in diabetes. Biochem Biophys Res Commun. 1990;173(3):932-9.

42. Arese P, Schwarzer E. Metabolic disorders: red cell alterations in diabetes mellitus. Berlin: Springer; Red cell membrane transport in health and disease 2003; 22:525-526.

43. Nwose EU, Jelinek HF, Richards RS, Kerr PG. Changes in the erythrocyte glutathione concentration in the course of diabetes mellitus. Redox Rep. 2006;11(3):99-104.
44. Ridker PM. High-sensitivity C-reactive protein: potential adjunct for global risk assessment in the primary prevention of cardiovascular disease. Circulation. 2001;103:1813-8.

45. Sjoholm A, Nystrom T. Endothelial inflammation in insulin resistance. Lancet. 2005;565:610-2.

46. Venugopal SK, Devaraj S, Jialal I. Effect of C-reactive protein on vascular cells: evidence for a proinflammatory, proatherogenic role. Curr Opin Nephrol Hypertens. 2005;14:33-7.

47. Gimbrone MA Jr, Topper JN, Nagel T, Anderson KR, GarciaCardena G. Endothelial dysfunction, hemodynamic forces, and atherogenesis. Ann NY Acad Sci. 2000;902:230-9.

48. Vita JA, Treasure CB, Nabel EG, Mclenachan JM, Fish RD, Yeung AC, et al. Coronary vasomotor response to acetylcholine relates to risk factors for coronary artery disease. Circulation. 1990;81(2):491-7.

49. Marenberg ME, Risch N, Berkman LF, Floderus B, de Faire U. Genetic susceptibility to death from coronary heart disease in a study of twins. N Engl J Med. 1994;330:1041-6.

50. Gaeta G, De Michele M, Cuomo S, Guarini P, Fogila MC, Bond MG, et al. Arterial abnormalities in the offspring of patients with premature myocardial infarction. N Engl J Med. 2000;343:840-6.

51. Giugliano D, Ceriello A, Esposito K. Glucose metabolism and hyperglycemia. Am J Clin Nutr. 2008;87:217S-22S.

52. Williams S. The association of the Glu298Asp polymorphism of endothelial nitric oxide synthase (eNOS) with outcome after subarachnoid hemorrhage. Doris Duke Med Stud J. 2003-2004;3: 52-56.

53. Zanchi A, Moczulski DK, Hanna LS, Wantman M, Warram JH, Krolewski AS. Risk of advanced diabetic nephropathy in type 1 diabetes is associated with endothelial nitric oxide synthase gene polymorphism. Kidney Int. 2000;57:405-13.

54. Ahluwalia TS, Ahuja M, Rai TS, Kohli HS, Sud K, Bhansali A, et al. Endothelial nitric oxide synthase gene haplotypes and diabetic nephropathy among Asian Indians. Mol Cell Biochem. 2008;314:9-17.

55. Wang XL, Greco M, Sim AS, Duarte N, Wang J, Wilcken DE. Effect of CYP1A1 MspI polymorphism on cigarette smoking related coronary artery disease and diabetes. Atherosclerosis. 2002;162(2):391-7.

56. Baserga R. The contradictions of the insulin-like growth factor 1 receptor. Oncogene. 2000;19(49):5574-81.

57. Zang Y, Wat N, Stratton IM, Warren-Perry MG, Orho M, Groop L, et al. UKPDS19: heterogeneity in NIDDM: separate contribution of IRS-1 and beta3-adrenergic-receptor mutations to insulin resistance and obesity respectively with no evidence for glycogen synthase gene mutations. Diabetologia. 1996;39: $1505-11$.

58. Hribal ML, Federici M, Porzio O, Lauro D, Borboni P, Accili D, et al. The Gly3Arg972 amino acid polymorphism in insulin receptor substrate-1 affects glucose metabolism in skeletal muscle cells. J Clin Endocrinol Metab. 2000;85:2004-13.

59. Federici M, Hribal ML, Ranalli M, Marselli L, Porzio O, Lauro $\mathrm{R}$, et al. The common Arg972 polymorphism in insulin receptor substrate-1 causes apoptosis of human pancreatic islets. FASEB J. 2001;15:22-4.

60. Holzl B, Iglseder B, Stadlmayr A, Hedegger M, More E, Reiter R, et al. Intima media thickness of carotid arteries is reduced in heterozygous carriers of the Gly972Arg variant in the insulin receptor substrate-1 gene. Eur J Clin Invest. 2003;33(2):110-6.

61. Dhananjayan R, Malati T, Brindha G, Kutala VK. Association of family history of type 2 diabetes mellitus with markers of endothelial dysfunction in South Indian population. Indian $\mathbf{J}$ Biochem Biophys. 2013;50:93-8.

62. Schalkwijk CG, Stehouwer CDA. Vascular complications in diabetes mellitus: the role of endothelial dysfunction. Clin Sci. 2005;109:143-59. 